\title{
A case series of rituximab induced tuberculosis
}

\author{
Nehal Parikh${ }^{1}$, Dhaiwat Shukla ${ }^{2}$, Kanishka Uttam Chandani ${ }^{3 *}$, Sapan Pandya ${ }^{2}$, \\ Supriya Malhotra ${ }^{1}$, Pankaj Patel ${ }^{4}$
}

${ }^{1}$ Department of Pharmacology, Smt. NHL Municipal Medical College, Ahmedabad, Gujarat, India

${ }^{2}$ Department of Rheumatology, Sheth Vadilal Sarabhai General Hospital, Ahmedabad, Gujarat, India

${ }^{3}$ Intern, Smt. NHL Municipal Medical College, Ahmedabad, Gujarat, India

${ }^{4}$ Dean, Smt. NHL Municipal

Medical College, Ahmedabad,

Gujarat, India

Received: 12 October 2018

Accepted: 01 November 2018

\section{*Correspondence to:}

Dr. Kanishka Uttam Chandani, Email: kanishka.uttamchandani @gmail.com

Copyright: () the author(s), publisher and licensee Medip Academy. This is an openaccess article distributed under the terms of the Creative Commons Attribution NonCommercial License, which permits unrestricted noncommercial use, distribution, and reproduction in any medium, provided the original work is properly cited.

\begin{abstract}
Rituximab has a myriad of clinical uses, ranging from its disease modifying action in rheumatoid arthritis, to its role in chemotherapy for cancer. Being an anti CD20 monoclonal antibody, it controls inflammation by targeting peripheral B cells including those present in the synovium. The use of Rituximab is associated with some side effects such as cytopenias and increased risk of infections such as JC virus reactivation leading to multifocal encephalopathy. The role of Rituximab as an immunosuppressant has been established. However, its association with tuberculosis in endemic countries like India is yet to be understood well. The study was a cross sectional study of the two cases reported about the incidence of tuberculosis in patients receiving infusions of rituximab for rheumatoid diseases. These adverse drug reactions were reported to the nearest pharmacovigilance center through the Vigiflow portal of WHO and were assessed for their causality as per the WHO scale. A 45 year old male patient, a known case of Systemic Lupus Erythematosus, presented to a tertiary care hospital with high grade fever with chills and rigors after which he was diagnosed with pleural effusion due to tuberculosis. The patient was on immunosuppressants which included Rituximab, Mycophenolate Sodium, Prednisolone and Hydroxychloroquine. Rituximab was withdrawn and the remaining medications were continued as per the initial plan. A 19 year old male patient, a known case of dermatomyositis and dilated cardiomyopathy, presented to a tertiary care hospital with complaints of fever with chills and rigors, and breathlessness on exertion which was followed by the diagnosis of miliary tuberculosis. Earlier, the patient was on Rituximab, Cyclophosphamide, Hydroxychloroquine and Prednisolone. Plan of further infusions of Rituximab and Cyclophosphamide was terminated while the remaining medications were continued. Both the patients were put on anti tubercular therapy and are now improving. The association of bacterial infections like tuberculosis with the use of Rituximab is not well understood. However, Rituximab being an immunosuppressant can be considered to be related to this infection. In our case series we readdress this association through a literature review.
\end{abstract}

Keywords: Rituximab, Rheumatoid diseases, Tuberculosis

\section{INTRODUCTION}

Rituximab has a myriad of clinical uses, ranging from its disease modifying action in rheumatoid arthritis, antineutrophil cytoplasmic antibody (ANCA)-associated vasculitis, to its role in chemotherapy for cancer. Being an anti CD20 monoclonal antibody, it targets peripheral B cells including those present in the synovium. Thus, it controls the inflammation caused by these cells in rheumatoid arthritis. Moreover, it causes variable depletion of B cells in other sites such as lymphoid tissue and bone marrow and thus has been found to be effective 
in the control of B-cell driven disorders. ${ }^{1}$ It depletes B cells by several mechanisms, including mediation of antibodydependent cellular cytotoxicity, complement-dependent cytotoxicity, and B cell apoptosis. Also, it exerts its action by affecting the $\mathrm{B}$ cell antigen presentation ability, $\mathrm{B}$ cell production of cytokines, and $\mathrm{B}$ cell production of autoantibodies such as rheumatoid factor. ${ }^{1,2}$ Rituximab, once a chemotherapeutic agent for a number of different types of non-Hodgkin lymphoma and for CD20+ chronic lymphocytic leukemia, has now found its place in the field of rheumatology. Currently, it is used for the treatment (both label and off label) of a vast variety of immunological disorders such as systemic lupus erythematosus, antiphospholipid syndrome, pemphigus and cicatricial pemphigoid, immune thrombocytopenia, and even neurological disorders like myasthenia gravis. ${ }^{3}$

Rituximab is used in hospitals as it is given as an intravenous infusion, although a subcutaneous formulation is also being evaluated. There are two different intravenous dosing strategies $-375 \mathrm{mg} / \mathrm{m}^{2}$ weekly for four weeks (lymphoma protocol) and $1000 \mathrm{mg}$ fortnightly (independent of body weight) for two doses (rheumatoid arthritis protocol). For some conditions and in some hospitals, the fortnightly strategy is modified to a low-dose strategy which involves two $500 \mathrm{mg}$ doses given 1-2 weeks apart. $^{4}$

The use of Rituximab is associated with some side effects such as infusion reactions, cytopenias with lymphopenia being the most common, increased risk of infections, acute kidney injury due to acute tumor lysis syndrome. Additionally, it is associated with cardiac adverse events, respiratory adverse events such as interstitial lung disease, acute abdomen, neurological side effects including cerebrovascular accidents, JC virus reactivation leading to multifocal encephalopathy. Dermatological reactions including paraneoplastic pemphigus, lichenoid dermatitis, vesiculobullous dermatitis, Stevens-Johnson syndrome, and toxic epidermal necrolysis have been noted. ${ }^{5}$ However, like every drug, rituximab also has a few contraindications to its usage. It cannot be given in pregnancy as well as severe congestive heart failure (New York Heart Association Class IV). Known hypersensitivity to rituximab or other mouse-derived proteins is also a relative contraindication. ${ }^{3}$

India, being a tuberculosis endemic country has about 1763876 new cases reported every year, as reported by WHO. The role of Rituximab as an immunosuppressant has been established. However, its association with tuberculosis in endemic countries is yet to be understood well. Tuberculosis is a lesser known infection that is associated with the use of Rituximab. Thus, we would like to report this novel side effect in the following two cases.

\section{CASE REPORT}

The study was a cross sectional study of the two cases reported about the incidence of tuberculosis in patients receiving infusions of rituximab for rheumatoid diseases. These adverse drug reactions were reported to the nearest pharmacovigilance center. Further, they were reported through the Vigiflow portal of WHO. Causality assessment of these adverse drug reactions was performed according to the scale provided by the WHO. After the literature review, the association was critically assessed, and the results were formulated.

\section{Case 1}

A 45 year old male patient, a known case of SLE, presented to a tertiary care hospital with high grade fever with chills and rigors with the fever occurring more commonly in the evening hours. Furthermore, the patient elicited a history of systemic lupus erythematosus accompanied by Libman-Sacks endocarditis, myositis and a psychiatric illness. Upon performing further investigations, it was discovered that he was suffering from pleural effusion along with underlying consolidation suggestive of tuberculosis. The pleural fluid was tapped out and it turned out to be positive for adenosine deaminase (ADA). Also, the lab values were within normal limits. The patient was on immunosuppressants which included Rituximab, Mycophenolate Sodium, Prednisolone and Hydroxychloroquine. The patient had received two infusions of Rituximab 1000mg, a month apart, 4 months prior to presenting with TB. He was on Mycophenolate sodium 360mg QID, Hydroxychloroquine $300 \mathrm{mg}$ OD and Prednisolone 10mg OD. Other medications that he was on included Thyroxine 50 micrograms OD for 4 years, Calcium $50 \mathrm{~g}$ BD since 7 months, and Paracetamol 500mg SOS.

Rituximab was withdrawn, and the remaining medications were continued as per the initial plan. The patient was then started on Isoniazid, Rifampicin, Pyrazinamide, Ethambutol and Streptomycin as per the Revised National Tuberculosis Control Program by the Government of India and is now improving. This case was reported to the nearest ADR monitoring center and through Vigiflow (Vigiflow ID 2018-30888).

\section{Case 2}

A 19 year old male patient, a known case of dermatomyositis and dilated cardiomyopathy, presented to the rheumatology department of a tertiary care hospital with complaints of fever with chills and rigors along with breathlessness on exertion. He developed these symptoms 10 days after receiving an infusion of Rituximab. On investigating further, the CT scan and chest X-Ray revealed miliary tuberculosis which was confirmed by a positive test for acid fast bacilli in his sputum. Earlier, the patient was on immunosuppressants which comprised of Rituximab, Cyclophosphamide, Hydroxychloroquine and Prednisolone. He had received a slow IV infusion of Rituximab 500mg 10 days prior to presentation. He had also taken two intravenous infusions of cyclophosphamide $500 \mathrm{mg}$ with a gap of one month in between them. 
Additionally, the patient was on $20 \mathrm{mg}$ of Prednisolone which was continued even after the adverse event with no change in the dose. Other medications that the patient was taking included Ramipril $0.5 \mathrm{mg}$ OD and Hydroxychloroquine 300mg OD.

Plan of further infusions of Rituximab and Cyclophosphamide was terminated. The patient was put on anti tubercular therapy consisting if Isoniazid, Rifampicin, Pyrazinamide, Ethambutol and Streptomycin. The patient's condition is improving. This case was reported to the nearest ADR monitoring center and through Vigiflow (Vigiflow ID 2018-33676).

\section{DISCUSSION}

The introduction of immunosuppressants such as tumor necrosis factor- $\alpha$ (TNF) blocking agents to more effectively treat chronic inflammatory disease has been a major breakthrough. Because these agents modulate the immune system, not unexpectedly, a number of infectious adverse events have been observed. There is considerable evidence that links reactivation of latent tuberculosis to the use of immunosuppressants such as anti-TNF monoclonal antibody $(\mathrm{mAb})$ treatments, which appear to result in disruption of the granuloma that normally compartmentalizes but does not kill Mycobacterium tuberculosis during latent tuberculosis. This effect can be explained, in part, by directly neutralizing TNF, which plays a key role in tuberculosis immunity. ${ }^{6}$ A study conducted in Mexico showed that patients with rheumatoid arthritis receiving biologics would benefit from isoniazid prophylaxis. ${ }^{7}$

Tuberculosis invites grave morbidity and mortality especially in patients suffering from autoimmune diseases receiving immunosuppressants. Moreover, the use of Rituximab is limited owing to its cost. Thus, the side effects in a tuberculosis endemic country like India are not well understood. This case series aims to report this novel association of tuberculosis with Rituximab. A study that aimed to study the safety profile of rituximab in rheumatoid arthritis and establish a consensus on its use stated that in the clinical trial safety database, two cases of pulmonary tuberculosis have been reported; these appear to have been de novo infections (information from Roche). ${ }^{8}$

A study conducted in the Kingdom of Saudi Arabia shows a contrasting result that Rituximab has no correlation with the flaring up of tuberculosis or developing the disease in patients on Rituximab including those in endemic regions. ${ }^{9}$ Another study conducted in a TB endemic region in the past has shown that Rituximab has a tendency to cause interstitial lung disease, the findings of which mimic tuberculosis. ${ }^{10}$ However, in the cases studied in this research, the investigations confirmed the diagnosis of tuberculosis.
On causality assessment, these adverse drug reactions were analyzed according to the WHO causality assessment criteria and were labelled as possible. Since both the patients reported were on more than one immunosuppressant, the probability of tuberculosis could be due to the added effect of immunosuppression and not due to Rituximab alone. However, the patients had been administered rituximab recently which is why the association of this drug with the disease should be a clinical suspicion.

\section{CONCLUSION}

The association of bacterial infections like tuberculosis with the use of Rituximab is not well understood. However, Rituximab being an immunosuppressant can be considered to be related to this infection. In our case series, we readdress this association through a literature review.

\section{ACKNOWLEDGEMENTS}

Authors would like to thank the Dean of the college and the Superintendent who allowed them to conduct the research in the hospital premises. Authors also like to show their gratitude to the Department of Rheumatology for the endless support and guidance at every step of the project and Department of Pharmacology who provided insight and expertise that greatly assisted the research.

Funding: No funding sources

Conflict of interest: None declared

Ethical approval: Not required

\section{REFERENCES}

1. Pescovitz MD. Rituximab, an anti-CD20 monoclonal antibody: history and mechanism of action. Am J Transplant. 2006;6(5 Pt 1):859-66.

2. Leandro MJ, Cooper N, Cambridge G, Ehrensein MR, Edwards JC. Bone-marrow B-lineage cells in patients with rheumatoid arthritis following Rituximab therapy. Rheumatology (Oxford). 2007;46(1):29-36.

3. Randall KL. Rituximab in autoimmune diseases. Australian Prescriber. 2016;39(4):131-4.

4. Chay J, Donovan P, Cummins L, Kubler P, Pillans P. Experience with low-dose rituximab in off-label indications at two tertiary hospitals. Intern Med J. 2013;43:871-82.

5. Kasi PM, Tawbi HA, Oddis CV, Kulkarni HS. Clinical review: Serious adverse events associated with the use of Rituximab - a critical care perspective. Critical Care. 2012;16(4):231.

6. Keane J. TNF-blocking agents and tuberculosis: new drugs illuminate an old topic, Rheumatol. 1 June 2005;44(6):714-20.

7. Hernandez-Cruz B, Ponce-de-Leon-Rosales S, Sifuentes-Osornio J, Ponce-de-Leon-Gardu o A, DiazJouanen E. Tuberculosis prophylaxis in patients with steroid treatment and systemic rheumatic diseases, A 
case-control study. Clinical and experimental rheumatology. 1999 Jan 1;17:81-7.

8. Buch MH, Smolen JS, Betteridge N, Breedveld FC, Burmester G, Dörner T, et al. Updated consensus statement on the use of rituximab in patients with rheumatoid arthritis. Annals of the rheumatic diseases. 2011 Jun 1;70(6):909-20.

9. Alkadi A, Alduaiji N, Alrehaily A. Risk of tuberculosis reactivation with Rituximab therapy. International Journal of Health Sciences. 2017;11(2):41-4.
10. Yao CW, Liao WC, Tu CY, Chen HJ, Teseng GC, Yeh SP. Rituximab-induced pneumonitis mimicking miliary tuberculosis. European Respiratory Review. 2013 Dec 1;22(130):587-8.

Cite this article as: Parikh N, Shukla D, Chandani U, Pandya S, Malhotra S, Patel P. A case series of rituximab induced tuberculosis. Int J Basic Clin Pharmacol 2018;7:2468-71. 